\title{
Experimental status of LNU in B decays in LHCb
}

\author{
Sean Benson ${ }^{1 \star}$ on behalf of the LHCb collaboration
}

1 Nikhef National Institute for Subatomic Physics at Amsterdam, Netherlands

$\star$ sean.benson@cern.ch

Proceedings for the 15th International Workshop on Tau Lepton Physics, Amsterdam, The Netherlands, 24-28 September 2018

doi:10.21468/SciPostPhysProc. 1

\begin{abstract}
In the Standard Model, the three charged leptons are identical copies of each other, apart from mass differences. Experimental tests of this feature in semileptonic decays of b-hadrons are highly sensitive to New Physics particles which preferentially couple to the 2nd and 3rd generations of leptons. A review of the latest lepton universality tests in semileptonic $b \rightarrow c$ transitions at LHCb is reported.
\end{abstract}

(c) () () Copyright S. Benson et al. This work is licensed under the Creative Commons Attribution 4.0 International License.

Published by the SciPost Foundation.
Received 18-01-2019

Accepted 21-01-2019

Published 19-02-2019

doi:10.21468/SciPostPhysProc.1.011

\section{Introduction}

Lepton Flavour Universality (LFU) requires the equality of the couplings between the gauge bosons and each of the three families of leptons. In practise this means that branching fractions of decays involving different lepton families do not depend on lepton flavour but differ only by phase space and helicity-suppressed contributions. The Standard Model predicts LFU. Therefore experimental evidence of Lepton Flavour Non-Universality (LFNU) would be a clear sign of physics beyond the SM (BSM).

Semileptonic and rare decays of heavy hadrons are an excellent laboratory to test LFNU as all three generations may be tested. A large class of models extending the SM contains additional interactions that could violate LFU. One such example is an extended Higgs Sector, which could have a large effect on semitauonic decay rates through the coupling to new charged Higgs scalars [1]. As such, semileptonic decays of b-hadrons to the third generation provide a sensitive probe of BSM effects. In addition, also $\mathrm{b} \rightarrow$ sll transitions are an excellent LFNU probe due to their sensitivity to BSM theories involving leptoquarks [2,3] or Z' $[4,5]$ particles. Leptoquarks have also been predicted to have an impact on semileptonic decays [6].

A precise measurement of a $B$ decay to $\tau$ leptons is challenging at a hadron collider due to the large background from partially reconstructed $B$-hadron decays that appear similar to the signal topology. Moreover, the signal decay kinematics can not be fully constrained because of the presence of neutrinos in the final state. Ratios of $\mathcal{R}\left(D^{*}\right) \equiv \frac{\mathcal{B}\left(\bar{B}^{0} \rightarrow D^{*+} \tau^{-} \bar{\nu}_{\tau}\right)}{\mathcal{B}\left(\bar{B}^{0} \rightarrow D^{*+} \mu^{-} \bar{v}_{\mu}\right)}$ use different $\tau$ lepton decays to provide independent measurements. The HFLAV combination for $\mathcal{R}\left(D^{*}\right)$ versus $\mathcal{R}(D)$ is shown in Fig. 1 along with the SM prediction and individual measurements. 


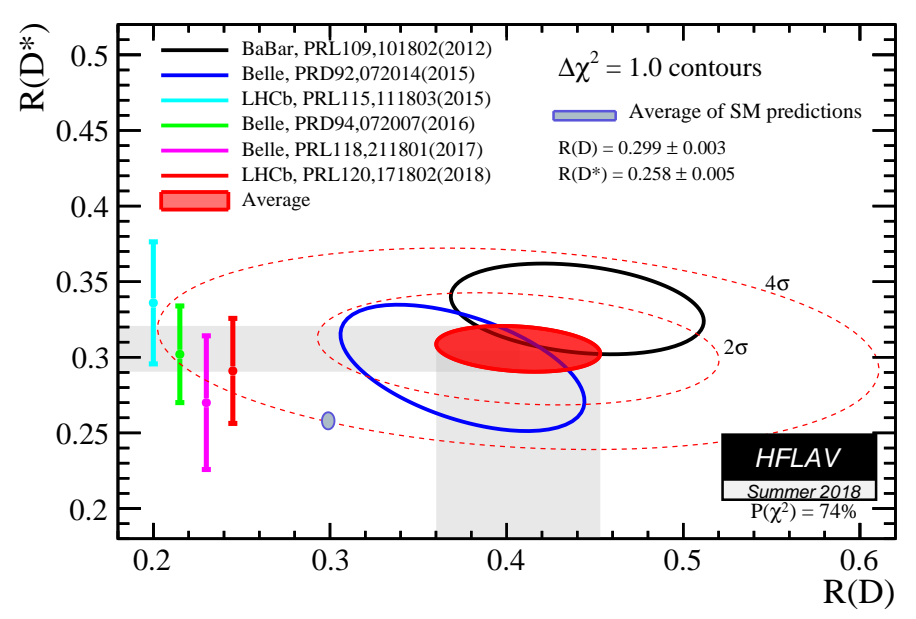

Figure 1: Individual measurements of $\mathcal{R}\left(D^{*}\right)$ and $\mathcal{R}(D)$ along with the combination and SM prediction [7].

Ratios of branching fractions represent a powerful test of LFNU due to the cancellation of theoretical uncertainites and experimental systematic uncertainties. In the following sections, the ratios involving semileptonic $\mathrm{b} \rightarrow c$ results obtained analysing the LHC Run 1 data collected by the LHCb experiment in 2011 and 2012 at a centre of mass energy of 7 and $8 \mathrm{TeV}$ are presented.

\section{Measurements of $\mathcal{R}\left(D^{*}\right)$}

\section{$2.1 \quad \bar{B}^{0} \rightarrow D^{*+} \tau^{-}\left(\rightarrow \mu^{-} v_{\tau} \bar{\nu}_{\mu}\right) \bar{\nu}_{\tau}$}

The muonic decay of the $\tau$ has an advantage that both the decay modes in the ratio $D^{*}$ contain the same visible final state, with one and three neutrinos visible in the final states, respectively. The measurement of the $\mathcal{R}\left(D^{*}\right)$ using the $\bar{B}^{0} \rightarrow D^{*+} \tau^{-}\left(\rightarrow \mu^{-} v_{\tau} \bar{v}_{\mu}\right) \bar{v}_{\tau}$ decay was performed with $3 \mathrm{fb}^{-1}$ of data collected at LHCb between 2011 and 2012 [8]. The $B$ momentum direction is determined from the unit vector to the $B$ decay vertex from the associated primary vertex. The component of the $B$ momentum along the beam axis is approximated using the relation $\left(p_{B}\right)_{z}=m_{B^{0}} / m_{\text {reco }}\left(p_{\text {reco }}\right)_{z}$, where $m_{B}^{0}$ is the known $B^{0}$ mass, $m_{\text {reco }}$ and $p_{\text {reco }}$ are the mass and momentum of the system of reconstructed particles. The rest-frame variables described above are then calculated using the resulting estimated $B$ four-momentum and the measured fourmomenta of the $\mu^{-}$and $D^{*+}$. The rest-frame variables are shown in simulation studies to have sufficient resolution to preserve the discriminating features of the original distributions. The candidates for both the signal and normalization channels are selected using a common procedure. The separation between signal and normalization channel is then achieved by exploiting the difference in the charged-lepton masses and the kinematic effect of the two extra neutrinos in the signal channel. The three kinematic variables used are: the muon energy $\left(E_{\mu}^{*}\right)$, the squared missing mass $\left(m_{\text {miss }}^{2}\right)$, and the squared four-momentum transfer to the lepton system $\left(q^{2}\right)$, which are evaluated in an approximated $B^{0}$ rest frame. The distributions of the variables are then fitted in a maximum likelihood fit, using template distributions taken from simulated data in addition to real data control samples. Several sources of background are taken into account, including partially reconstructed decays of $D^{*}$ and $D^{* *}$ mesons, decays to pairs of charm hadrons, and candidates combinatorial in nature. The $m_{\mathrm{miss}}^{2}$ and $E_{\mu}^{*}$ distri- 

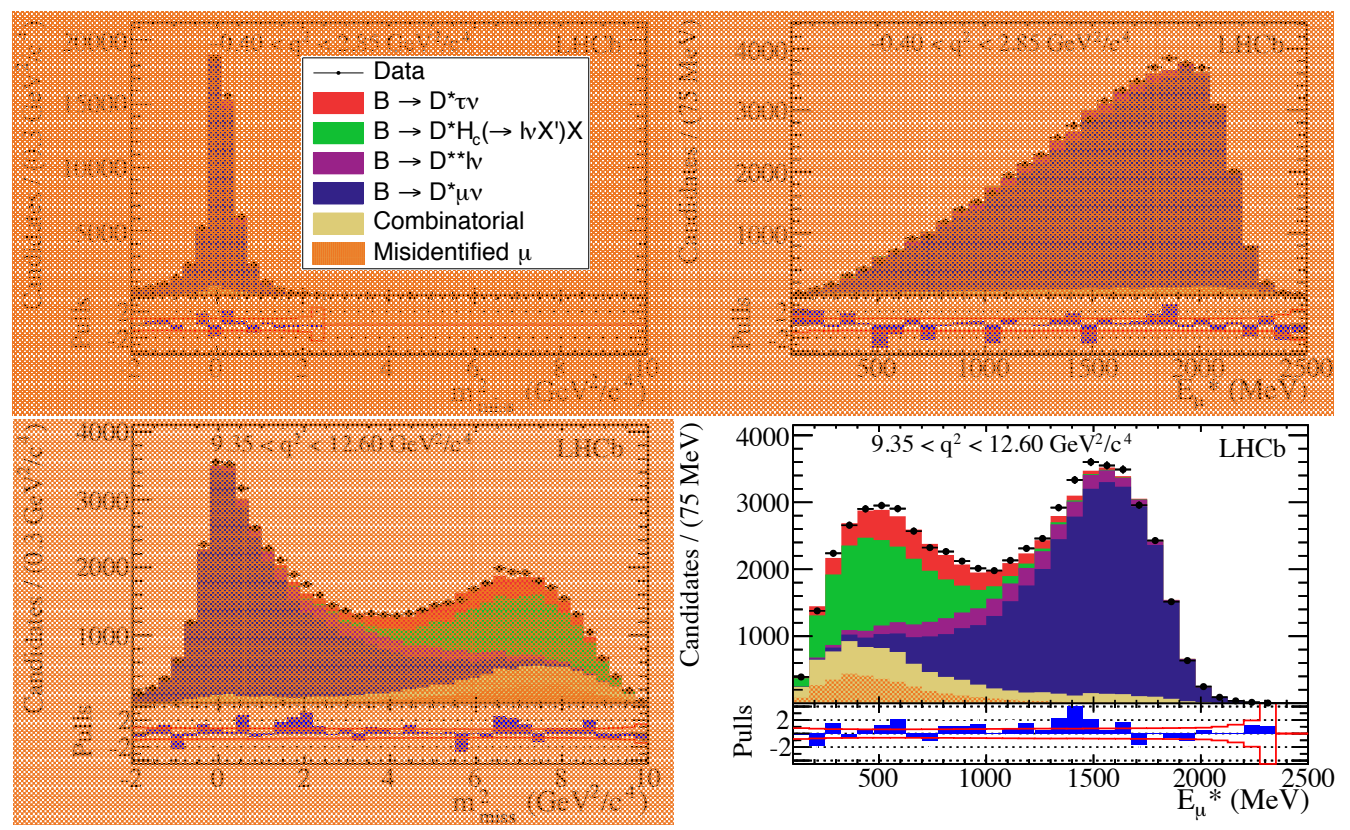

Figure 2: Distributions of $m_{\text {miss }}^{2}$ (left) and $E_{\mu}^{*}$ (right) of the low- (top) and high- (bottom) $q^{2}$ bins of the signal data, overlaid with projections of the fit model with all normalization and shape parameters at their best-fit values. Below each panel differences between the data and fit are shown, normalized by the Poisson uncertainty in the data. The bands give the $1 \sigma$ template uncertainties [8].

butions of fully-selected candidates are shown in Fig. 2 for the lowest and highest $q^{2}$ bins. The yields extracted by the fit are used to make a measurement of $\mathcal{R}\left(D^{*}\right)$, which is found to be $0.336 \pm 0.027 \pm 0.030$, where the first uncertainty is statistical and the second systematic in nature. The result is the first measurement of b-hadron decays to $\tau$ leptons at a hadron collider and represents a $2.1 \sigma$ deviation from the SM prediction.

Systematic uncertainties are dominated by the size of simulated and real data control samples, which will decrease as more data is simulated and collected, respectively.

\section{$2.2 \quad \bar{B}^{0} \rightarrow D^{*+} \tau^{-}\left(\rightarrow \pi^{+} \pi^{-} \pi^{-} \nu_{\tau}\right) \bar{\nu}_{\tau}$}

A more recent measurement of $\mathcal{R}\left(D^{*}\right)$ is made using the hadronic decay of the $\tau^{-} \rightarrow \pi^{+} \pi^{-} \pi^{-} v_{\tau}$ [9], using the Run 1 dataset. The $D^{*}$ meson is reconstructed through the $D^{*-} \rightarrow D^{-}\left(\rightarrow \mathrm{K}^{+} \pi^{-}\right) \pi^{-}$ decay chain. The analysis extracts the ratio $\mathcal{K}\left(D^{*}\right)$ defined as

$$
\begin{aligned}
\mathcal{K}\left(D^{*}\right) & =\frac{\mathcal{B}\left(B^{0} \rightarrow D^{*+} \tau^{-} \bar{v}_{\tau}\right)}{\mathcal{B}\left(B^{0} \rightarrow D^{*-} 3 \pi\right)} \\
& =\frac{N_{B^{0} \rightarrow D^{*+} \tau^{-} \bar{v}_{\tau}}}{N_{B^{0} \rightarrow D^{*-}-3 \pi}} \frac{\varepsilon_{B^{0} \rightarrow D^{*-} 3 \pi}}{\varepsilon_{B^{0} \rightarrow D^{*+} \tau^{-} \bar{v}_{\tau}}} \frac{1}{\mathcal{B}\left(\tau \rightarrow 3 \pi \bar{v}_{\tau}\right)},
\end{aligned}
$$

where $N_{i}$ and $\varepsilon_{i}$ are the yield and the selection efficiency, respectively. The ratio $\mathcal{R}\left(D^{*}\right)$ is subsequently obtained with the relation

$$
\mathcal{R}\left(D^{*}\right)=\mathcal{K}\left(D^{*}\right) \frac{\mathcal{B}\left(B^{0} \rightarrow D^{*} 3 \pi\right)}{\mathcal{B}\left(B^{0} \rightarrow D^{*} \mu v_{\mu}\right)}
$$

where the branching ratios are taken from external inputs [10]. The main background contribution is due to the inclusive decays of b-hadrons to the $D^{*} 3 \pi X$ final state. A good suppression of such background is obtained by requiring the $\tau$ decay vertex is displaced from the $B$ decay 
vertex along the beam direction with a $4 \sigma$ significance. Candidates that survive the decay length significance requirement are mainly double-charmed $B$ decays of type $B \rightarrow D^{*+} D^{*-} X$. In order to discriminate this background from the signal decay, a Boosted Decision Tree (BDT) is trained, using isolation variables (describing the separation of signal decay particles from other decays), kinematic variables, and variables which exploit the differences between the resonant structures of the 3-pion system from signal and $D_{s}^{+}$background decays.

The yield of the normalization mode is determined by fitting the invariant mass of $D^{*-} 3 \pi$ system. The signal yield is obtained from a three-dimensional extended maximum likelihood fit to the BDT output, the $\tau$ decay time, and the $q^{2}$. The results of the fit are shown in Fig. 3. The shapes of the various components that are taken from templates of simulated samples and corrected according to data control samples.
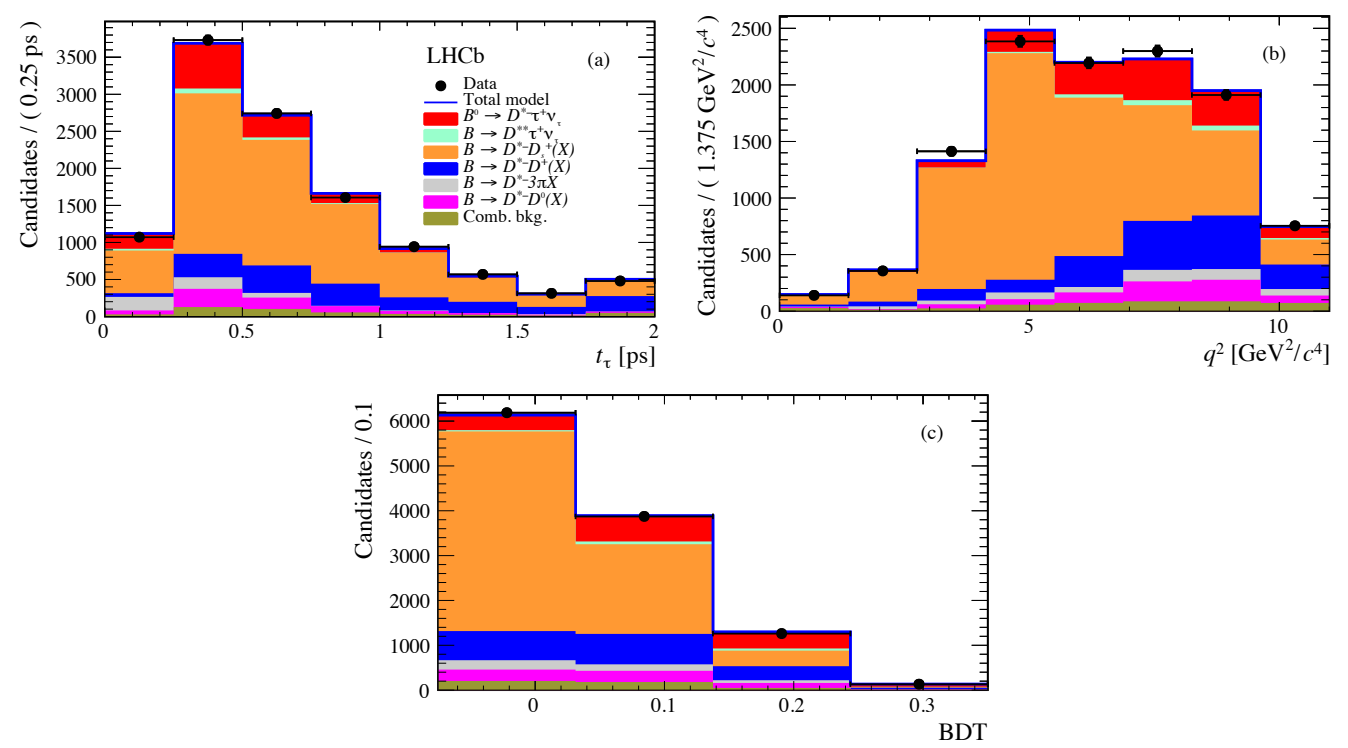

Figure 3: Projections of the three-dimensional fit on the (a) $3 \pi$ decay time, (b) $q^{2}$ and (c) BDT output distributions. The fit components are described in the legend [9].

The measured value of $\mathcal{K}\left(D^{*}\right)$ is found to be $1.97 \pm 0.13 \pm 0.18$ where the first uncertainty is statistical and the second systematic. Using external inputs [10], the value of $\mathcal{R}\left(D^{*}\right)$ is calculated to be $0.291 \pm 0.019 \pm 0.026 \pm 0.013$ where the third uncertainty originates from the limited knowledge of branching fraction of the normalization channel. The measurement of $\mathcal{R}\left(D^{*}\right)$ is one of the most precise and contains the smallest statistical error. It is compatible with the SM prediction within $1 \sigma$.

\section{Measurement of $\mathcal{R}(\mathrm{J} / \psi)$}

The measurement of $\mathcal{R}(\mathrm{J} / \psi)$ probes similar physics as that of $\mathcal{R}\left(D^{*}\right)$, with similar SM predictions [11]. The ratio measured in the LHCb analysis is the same as that in Ref. [8], with the $D^{*}$ replaced by a $\mathrm{J} / \psi$ and the $B^{0}$ meson is replaced by a $B_{c}^{+}$meson. The analysis uses the full Run 1 dataset of $3 \mathrm{fb}^{-1}$ [12].

A binned maximum likelihood fit is performed to determine the signal and normalization yields. The components in the fit are taken from simulated templates. The three dimensions of the templates consist of $m_{\text {miss }}^{2}$, the $B_{c}$ decay time, along with a categorical quantity $Z$, representing eight bins in $\left(E_{\mu}^{*}, q^{2}\right)$. The values $0-3$ of $Z$ correspond to bins where $q^{2}<7.15 \mathrm{GeV}^{2} / c^{4}$ and $E_{\mu}^{*}$ is divided with thresholds at $[0.68,1.15,1.64] \mathrm{GeV}$. The values 
4-7 correspond to bins with the same $E_{\mu}^{*}$ ranges, but where $q^{2} \geq 7.15 \mathrm{GeV}^{2} / c^{4}$. The fit result to the three template dimensions are shown in Fig. 4. The fit results in a measured value of
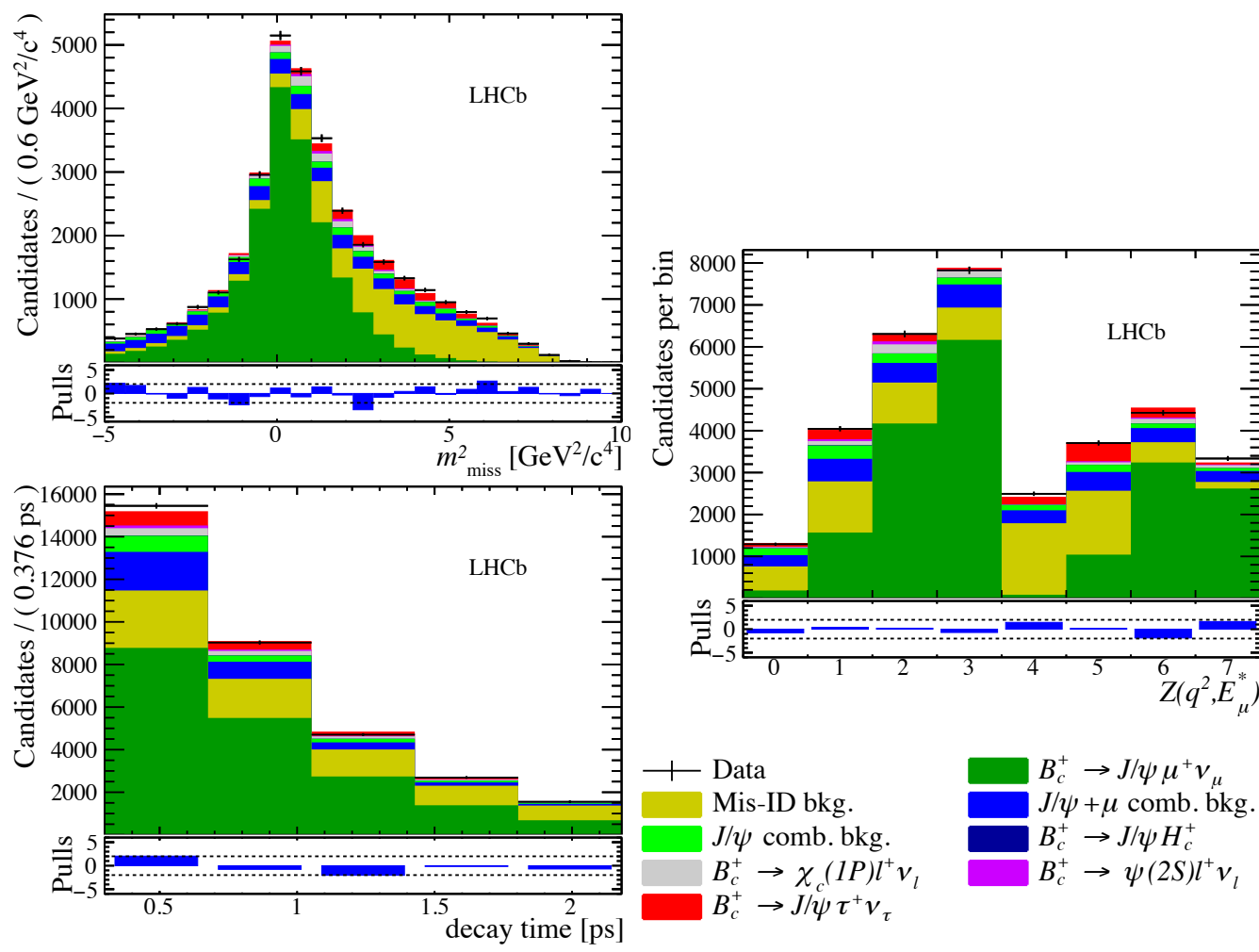

Figure 4: Distributions of (top-left) $m_{\text {miss }}^{2}$, (bottom-left) decay time, and (right) $Z$ of the signal data, overlaid with projections of the fit model with all normalization and shape parameters at their best-fit values. Below each panel differences between the data and fit are shown, normalized by the Poisson uncertainty in the data; the dashed lines are at the values $\pm 2[12]$.

$\mathcal{R}(\mathrm{J} / \psi)=0.71 \pm 0.17 \pm 0.18$, where the first uncertainty is statistical and the second systematic in nature. The unknown form factors used in the generation of the simulated samples gives rise to the largest systematic uncertainty. The result is compatible with the SM prediction at the level of $2 \sigma$.

\section{Summary and prospects}

Three measurements of LFU using semi-leptonic decays to $\tau$ leptons have been presented using $3 \mathrm{fb}^{-1}$ of Run 1 data collected between 2011 and 2012. They consist of the first measurement of $\mathcal{R}\left(D^{*}\right)$ at a hadron collider, the most accurate single measurement of $\mathcal{R}\left(D^{*}\right)$, along with the first measurement of $\mathcal{R}(\mathrm{J} / \psi)$. The current tension with SM predictions is approximately $3.8 \sigma$ [7]. The LHCb experiment has, at the time of writing, collected approximately $9 \mathrm{fb}^{-1}$ of data. Updates to the analyses presented here are currently underway, which will help to provide even more accurate measurements, as the systematic uncertainties will decrease with larger simulation and control data samples.

In 2021, the upgraded LHCb experiment will begin collecting data [13]. After a single year of data taking with the LHCb upgrade, the combined dataset of LHCb will be powerful enough to reduce uncertainties to $25 \%$ of the values reported in these proceedings [14]. If the luminosity of the LHCb Phase II upgrade is realised, $10 \%$ of the uncertainties in these is achievable. Uncertainties as a function of luminosity are shown in Fig. 5. 


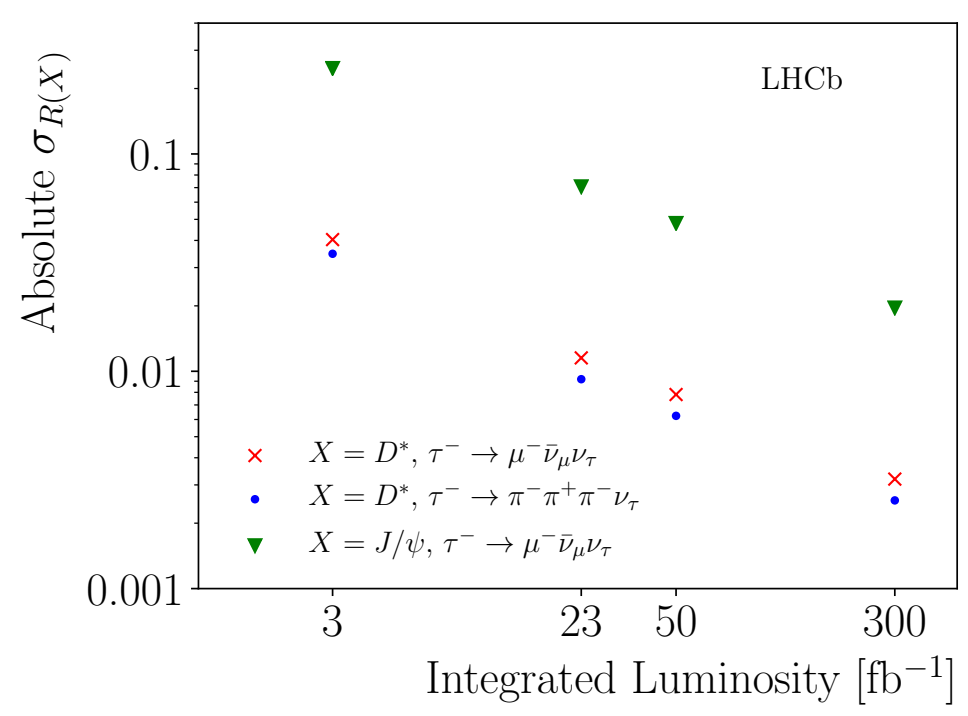

Figure 5: Prospects for different LFNU tests as more data is collected with at LHCb [14].

\section{References}

[1] M. Tanaka, Charged Higgs effects on exclusive semi-tauonic B decays, Z. Phys. C - Particles and Fields 67, 321 (1995), doi:10.1007/BF01571294.

[2] D. Bečirević, S. Fajfer, N. Košnik and O. Sumensari, Leptoquark model to explain the B-physics anomalies, $R_{K}$ and $R_{D}$, Phys. Rev. D 94, 115021 (2016), doi:10.1103/PhysRevD.94.115021.

[3] A. Crivellin, D. Müller and T. Ota, Simultaneous explanation of $R\left(D^{(*)}\right)$ and $b \rightarrow s \mu^{+} \mu^{-}$: the last scalar leptoquarks standing, J. High Energ. Phys. 09, 040 (2017), doi:10.1007/JHEP09(2017)040.

[4] W. Altmannshofer, S. Gori, S. Profumo and F. S. Queiroz, Explaining dark matter and $B$ decay anomalies with an $L_{\mu}-L_{\tau}$ model, J. High Energ. Phys. 12, 106 (2016), doi:10.1007/JHEP12(2016)106.

[5] A. J. Buras and J. Girrbach, Left-handed $Z^{\prime}$ and $Z$ FCNC quark couplings facing new $b \rightarrow s \mu^{+} \mu^{-}$data, J. High Energ. Phys. 12, 009 (2013), doi:10.1007/JHEP12(2013)009.

[6] D. Buttazzo, A. Greljo, G. Isidori and D. Marzocca, B-physics anomalies: a guide to combined explanations, J. High Energ. Phys. 11, 044 (2017), doi:10.1007/JHEP11(2017)044.

[7] Y. Amhis et al., Averages of b-hadron, c-hadron, and $\tau$-lepton properties as of summer 2016, Eur. Phys. J. C 77, 895 (2017), doi:10.1140/epjc/s10052-017-5058-4.

[8] R. Aaij et al., Measurement of the ratio of branching fractions $\mathcal{B}\left(\bar{B}^{0} \rightarrow D^{*+} \tau^{-} \bar{v}_{\tau}\right) / \mathcal{B}\left(\bar{B}^{0} \rightarrow D^{*+} \mu^{-} \bar{v}_{\mu}\right)$, Phys. Rev. Lett. 115, 111803 (2015), doi:10.1103/PhysRevLett.115.111803, [Publisher's note: Phys. Rev. Lett. 115, 159901(2015), doi:10.1103/PhysRevLett.115.159901]

[9] R. Aaij et al., Test of lepton flavor universality by the measurement of the $B^{0} \rightarrow D^{*-} \tau^{+} v_{\tau}$ branching fraction using three-prong $\tau$ decays, Phys. Rev. D 97, 072013 (2018), doi:10.1103/PhysRevD.97.072013. 
[10] M. Tanabashi et al., Review of particle physics, Phys. Rev. D 98, 030001 (2018), doi:10.1103/PhysRevD.98.030001.

[11] E. Hernández, J. Nieves and J. M. Verde-Velasco, Study of exclusive semileptonic and nonleptonic decays of $B_{c}$ - in a nonrelativistic quark model, Phys. Rev. D 74, 074008 (2006), doi:10.1103/PhysRevD.74.074008.

[12] R. Aaij et al., Measurement of the ratio of branching fractions $\mathcal{B}\left(B_{c}^{+} \rightarrow J / \psi \tau^{+} v_{\tau}\right) / \mathcal{B}\left(B_{c}^{+} \rightarrow J / \psi \mu^{+} v_{\mu}\right)$, Phys. Rev. Lett. 120, 121801 (2018), doi:10.1103/PhysRevLett.120.121801.

[13] LHCb Collaboration, Computing Model of the Upgrade LHCb experiment, Tech. Rep. CERNLHCC-2018-014. LHCB-TDR-018 (2018), http://cds.cern.ch/record/2319756.

[14] I. Bediaga et al., Physics case for an LHCb Upgrade II - Opportunities in flavour physics, and beyond, in the HL-LHC era (2018), arXiv:1808.08865. 